\title{
Pathological basal ganglia calcification in a patient with pseudohypoparathyroidism
}

\author{
L Sweidan, MB ChB \\ Division of Radiology, Department of Radiation Sciences, University of the \\ Witwatersrand, Johannesburg
}

Bilateral symmetrical calcification of the basal ganglia is a frequently encountered incidental finding on CT scan. The aetiology of adult basal ganglia calcification ranges from a physiological type secondary to aging, which may be regarded as a normal variant, to the less typical pathological causes. Physiological basal ganglia calcification is usually punctate, localised in the globus pallidus and of no clinical significance. If accompanied by other calcification one should then consider an underlying pathological condition. Endocrine diosorders, e.g. hypoparathyroidism, pseudohypoparathyroidism and hyperparathyroidism are the more commonly recognised pathological causes of basal ganglia calcification. ${ }^{1,2}$

The calcification may be dense and its distribution frequently involves the caudate and lentiform nuclei, thalami, dentate nuclei of the cerebellum and subcortical regions (Figs 1 and 2). The above non-contrast axial CT in a 60-year-old patient with known pseudohypoparathyroidism demonstrates an example thereof.

1. Oliveira JR, Spiteri E, Sobrido MJ, et al. Genetic heterogeneity in familial idiopathic basal ganglia calcification (Fahr disease). Neurology 2004; 63: 2165-2167.

2. Verulashvili IV, Glonti LSH, Maniia MN, et al. Basal ganglia calcification: clinical manifestations and diagnostic evaluation. Georgian Med News 2006; 140: 39-43.

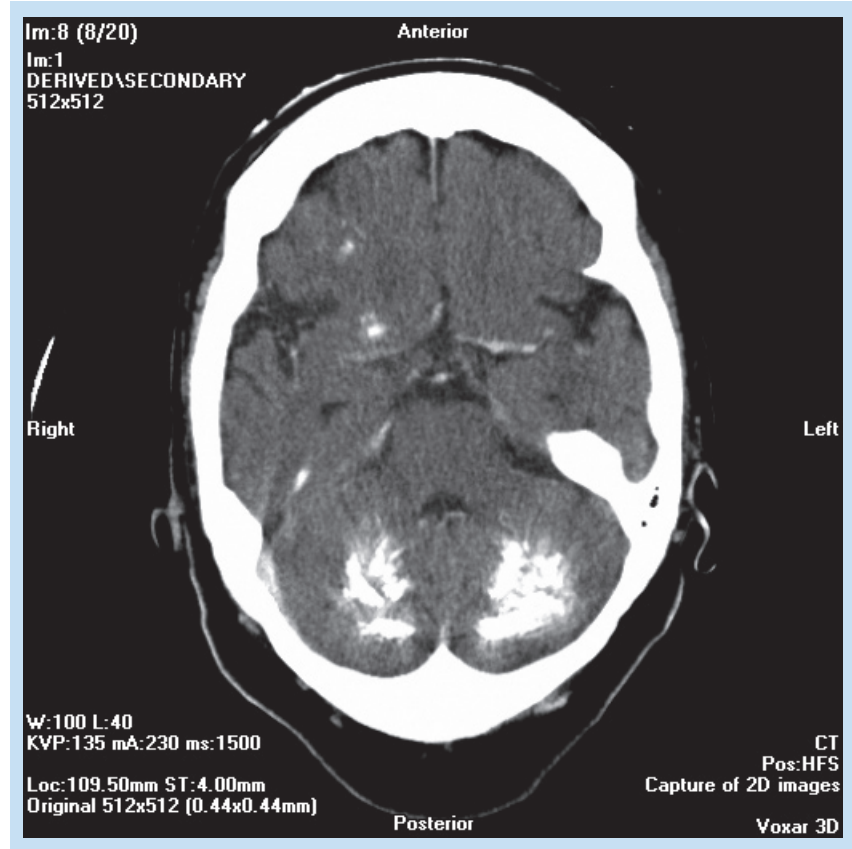

Fig. 1. Dense calcification of the dentate nuclei bilaterally.

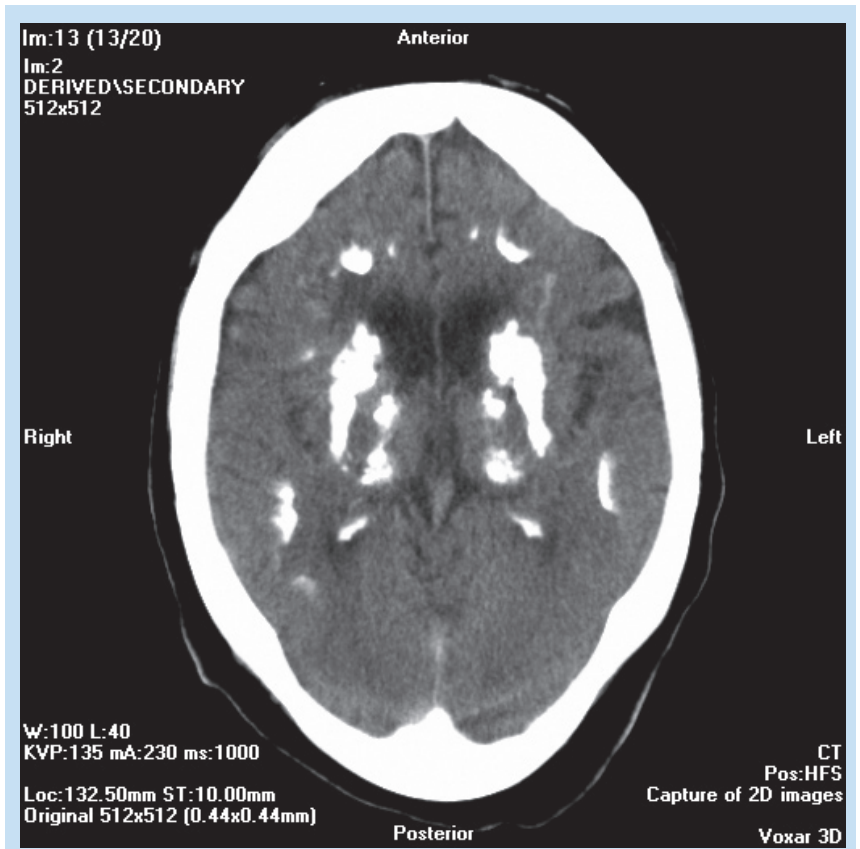

Fig. 2. Dense calcification of the basal ganglia, thalami, nuclei and subcortical white matter. 